\title{
Minimum-Fuel Aeroassisted Coplanar Orbit Transfer Using Lift-Modulation
}

\author{
Kenneth D. Mease* \\ Jet Propulsion Laboratory, California Institute of Technology, Pasadena, California \\ and \\ Nguyen X. Vinh $\dagger$ \\ University of Michigan, Ann Arbor, Michigan
}

\begin{abstract}
Minimum-fuel trajectories and lift controls are computed for aeroassisted coplanar transfers from high orbit to low orbit. The optimal aeroassisted transfer requires less fuel than the all-propulsive Hohmann transfer for a wide range of high orbit to low orbit transfers. The optimal control program for the atmospheric portion of the transfer is to fly at maximum positive lift-to-drag ratio $(L / D)$ initially to recover from the downward plunge, and then to fly at negative $L / D$ to level off the flight in such a way that the vehicle skips out of the atmosphere with a flight path angle near zero degrees. To avoid excessive heating rates the vehicle flies initially at high angle of attack in order to slow down higher in the atmosphere. This allows the subsequent recovery from the downward plunge, using the maximum positive $L / D$, to take place at a higher altitude, where the atmosphere is less dense.
\end{abstract}

\section{Nomenclature}

$C_{D}$

$C_{D_{0}}$

$C_{L}$

$C_{L}^{*}$

$E^{*}$

$F$

$g$

GEO

$h$

$H$

$H_{R}=$ convective heating rate for $1 \mathrm{~m}$ sphere

$\mathrm{HEO}=$ high Earth orbit

$K=$ coefficient in parabolic drag polar

$L / D=$ lift-to-drag ratio

LEO = low Earth orbit

$m \quad=$ vehicle mass

OTV = Orbital Transfer Vehicle

$p_{j} \quad=$ adjoint variable associated with state $j$

$r \quad=$ radial distance from Earth's center

$r_{1}=$ radius of $\mathrm{HEO}$

$r_{2} \quad=$ radius of $\mathrm{LEO}$

$R \quad=$ radius of spherical atmosphere

$S \quad=$ effective vehicle surface area normal to velocity

vector

$=V / \sqrt{\mu / R}$

$=$ inertial speed

$=r_{i} / R$

$=$ inertial flight path angle

$=\rho / \rho_{o}$

$=\Delta V_{i} / \sqrt{\mu / R}$

$\Delta v_{i}$

=impulsive change in $V$

Presented as Paper 83-2094 at the AIAA Atmospheric Flight Mechanics Conference, Gatlinburg, Tenn., Aug. 15-17, 1983; received Sept. 14, 1983; revision received Feb. 24, 1984. Copyright (C) American Institute of Aeronautics and Astronautics, Inc., 1983. All rights reserved.

*Member Technical Staff, Navigation Systems Section. Member AIAA.

†Professor, Aerospace Engineering.

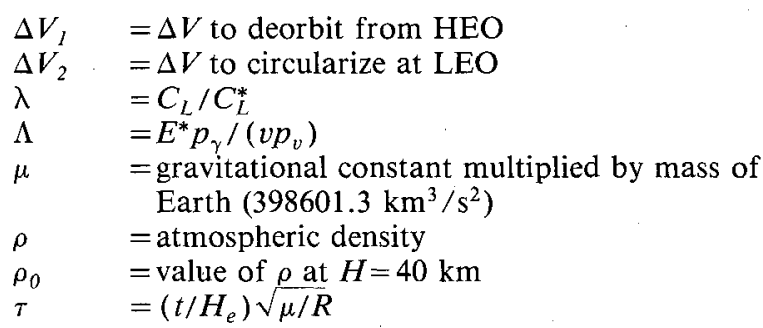

Subscripts

$e \quad=$ value at atmospheric entry

$f \quad=$ value at atmospheric exit

$p \quad=$ value at hypothetical perigee of transfer orbit from $\mathrm{HEO}$ to atmosphere

\section{Introduction}

$\mathbf{W}$ HEN orbital transfer is required and there is an atmosphere-bearing celestial body in the vicinity, it may be advantageous to utilize aerodynamic force in effecting the transfer. In this paper, we present an investigation of aeroassisted coplanar transfer from a circular orbit of radius $r_{l}$ to a concentric circular obit of radius $r_{2}$, where $r_{l}$ is greater than $r_{2}$ (Fig. 1). We will consider the orbits to be about the Earth; however much of the analysis is more generally applicable. Our assumptions are as follows. The vehicle has a lifting configuration; and the lift can be modulated by varying the angle of attack. Lift modulation is the sole means of controlling the flight path in the atmosphere, propulsion being used only outside the atmosphere. The vehicle has a high-thrust propulsion system so that applications of the thrust can be considered to produce impulsive velocity changes ( $\left.\Delta V^{\prime} s\right)$ and the fuel consumption for an orbital transfer is thus indicated by the characteristic velocity, the sum of the $\Delta V$ 's needed to effect the transfer. The transfer must involve only a single atmospheric pass. And finally, the atmospheric properties, the vehicle's aerodynamic properties, the equations of motion, and the initial position and velocity of the vehicle are all known precisely.

Under these assumptions, we determine the minimum-fuel aeroassisted transfer, the fuel requirements of which are then 
compared to those of the minimum-fuel, all-propulsive transfer. The characteristics of the minimum-fuel trajectory during the atmospheric portion of the aeroassisted transfer are examined in detail. In addition, the effect of a vehicle heating constraint on the atmospheric trajectory is determined.

The motivation for this study stems from the current interest in an orbital transfer. vehicle (OTV). ${ }^{1,2}$ This vehicle would transfer a spacecraft from a space shuttle to a higher and/or a different inclination orbit. The vehicle would then return, after delivering its cargo, to rendezvous with either a shuttle or a space operations center. The OTV maneuvers which could benefit potentially from aeroassist are the orbital plane change and the transfer from high Earth orbit (HEO) to low Earth orbit (LEO). The present study concerns only the latter.

The basic sequence of events for the aeroassisted HEO to LEO coplanar orbit transfer is as follows. Referring to Fig. 1, the transfer begins with an in-plane tangential retroburn $\left(\Delta V_{l}\right)$ at $\mathrm{HEO}$ which injects the vehicle into an elliptical transfer orbit with a hypothetical target perigee inside the atmosphere. At point $E$, the vehicle enters the atmosphere. As the vehicle flies through the atmosphere, some of its kinetic energy is converted to heat, and, consequently upon skipping out of the atmosphere (at point $F$ ), the apogee of the orbit is decreased to the distance $r_{2}$. Finally, at the new apogee, a second in-plane tangential burn $\left(\Delta V_{2}\right)$ is executed to circularize and thereby achieve the desired LEO. The minimumfuel aeroassisted transfer is that which has the minimum characteristic velocity, $\Delta V_{1}+\Delta V_{2}$. The flight path for the minimum-fuel transfer is effected by the $\Delta V_{I}$ magnitude, which controls the atmospheric entry, and the lift coefficient as a function of time during the atmospheric flight, which controls the exit and hence determines the required $\Delta V_{2}$.

\section{Analytic Solution for an Idealized Optimal Transfer}

Consider an aeroassisted HEO to LEO transfer which proceeds as follows. Referring to Fig. 1, a tangential retroburn $\Delta V_{I}$ at $\mathrm{HEO}$ injects the vehicle into an elliptical transfer orbit with perigee at the distance $R$. When the vehicle is at perigee, its lifting capability (in this case, negative lift) is employed to effect flight along the boundary of the atmosphere (i.e., along a circular orbit of radius $R$ ). Flight along the boundary is continued until sufficient velocity has been depleted (by atmospheric drag) such that upon reducing the lift to zero, the vehicle ascends on an elliptical orbit to an apogee at $r_{2}$. Finally, at $r_{2}$, a tangential circularizing burn, $\Delta V_{2}$, is executed to achieve the desired LEO. The idealizations here are 1) that the atmospheric density at $R$ is sufficient to

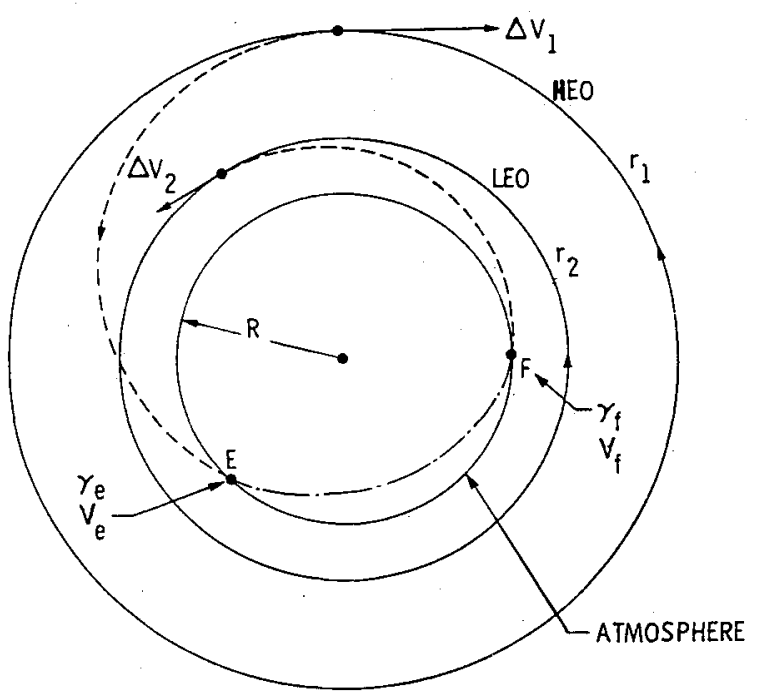

Fig. 1 Aeroassisted coplanar orbit transfer. generate enough drag to slow the vehicle in a reasonable amount of time, and 2) that the vehicle has sufficient lift to maintain flight along the atmospheric boundary.

The characteristic velocity of this idealized transfer is now compared with that of any realistic aeroassisted transfer. A realistic transfer would require a larger $\Delta V_{l}$ to ensure sufficient penetration into the atmosphere such that the required velocity is depleted before skipping back out, given the limited lifting capability of the vehicle. Thus the $\Delta V_{I}$ for the idealized transfer is a lower bound for aeroassisted transfers. Secondly, for the one-impulse transfer from atmospheric exit to LEO, exit with a flight path angle of zero degrees $\left(\gamma_{f}=0 \mathrm{deg}\right)$ into an elliptical transfer orbit, tangent to LEO at apogee, leads to the minimum circularizing $\Delta V_{2}$. The corresponding exit speed

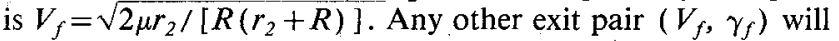
lead to a higher $\Delta V_{2}$. Consequently, the characteristic velocity, $\Delta V_{I}+\Delta V_{2}$, for this idealized aeroassisted transfer is a lower bound for the characteristic velocity of any realistic aeroassited transfer.

An analytic expression for this lower bound can be derived Let

$$
\alpha_{1}=r_{1} / R, \alpha_{2}=r_{2} / R, \text { and } \Delta v_{i}=\Delta V_{i} / \sqrt{\mu / R}
$$

The elliptical grazing trajectory requires an impulse

$$
\Delta v_{l}=\sqrt{1 / \alpha_{l}}-\sqrt{2 /\left[\alpha_{l}\left(\alpha_{l}+1\right)\right]}
$$

The second impulse used to circularize the orbit at $r_{2}$ is

$$
\Delta v_{2}=\sqrt{1 / \alpha_{2}}-\sqrt{2 /\left[\alpha_{2}\left(\alpha_{2}+1\right)\right]}
$$

Thus the total characteristic velocity for the idealized aeroassisted transfer is

$$
\Delta v_{A}=\Delta v_{1}+\Delta v_{2}
$$

Compare this to the characteristic velocity for the minimumfuel, all propulsive transfer, the Hohmann transfer, which is

$$
\begin{aligned}
\Delta v_{H} & =\sqrt{1 / \alpha_{1}-\sqrt{2 \alpha_{2} /\left[\alpha_{l}\left(a_{l}+\alpha_{2}\right)\right]}} \\
& +\sqrt{2 \alpha_{1} /\left[\alpha_{2}\left(\kappa_{l}+\alpha_{2}\right)\right]}-\sqrt{I / \alpha_{2}}
\end{aligned}
$$

The curve plotted in Fig. 2 represents pairs $\left(\alpha_{1}, \alpha_{2}\right)$ for which $\Delta v_{A}=\Delta v_{H}$. For pairs below the curve, $\Delta v_{A}<\Delta v_{H}$, i.e., the idealized aeroassisted transfer requires less fuel. For example, idealized aeroassisted transfer from geostationary orbit to LEO requires less fuel than the Hohmann transfer, if the LEO radius, $r_{2}$, is less than about $12,000 \mathrm{~km}$. In the case where $r_{2}=6728 \mathrm{~km}$ (a typical shuttle orbit radius), the characteristic velocity, for the transfer from GEO is $1.55 \mathrm{~km} / \mathrm{s}$ for the aeroassisted mode, as compared to $3.87 \mathrm{~km} / \mathrm{s}$ for the allpropulsive mode.

It should be noted that in the case of the aeroassisted transfer, the one-impulsede orbit to atmospheric entry and the oneimpulse LEO insertion following atmospheric exit are not always the optimal modes. As has been shown in Ref. 3, for the general case of deorbit from elliptical orbit, the optimal mode can be one-impulse or two-impulse; or it can be via a parabolic orbit (or more practically via an elongated ellipse). In the case of parabolic deorbit, an impulse is applied tangentially at the perigee to send the vehicle into a parabolic orbit. Then at a large distance, an infinitesimal impulse is applied to return the vehicle for entry at any prescrbed entry angle. For a circular orbit, the characteristic velocity for this mode is $\Delta v_{p}=\sqrt{2} / \alpha_{1}-\sqrt{1 / \alpha_{l}}$. With respect to the characteristic velocity for the one-impulse mode, Eq. (1), the parabolic mode is optimal whenever $\alpha_{l}>2(\sqrt{2}+1)=4.828$. Hence, optimally it should be used in the case of return from GEO. However, in this case, the saving in the characteristic velocity is only $21 \mathrm{~m} / \mathrm{s}$. Since the saving is small, while the 
time required to complete the deorbit is greatly increased, the parabolic mode shall not be further considered.

The two-impulse mode of deorbit can be optimal for a nonzero entry angle; but it can be shown that when it is, the saving in characteristic velocity as compared to the oneimpulse mode is of the order of $\left(\gamma_{e}\right) .{ }^{1}$ With regard to the oneimpulse LEO insertion following atmospheric exit, if the apogee of the post-exit orbit is exactly at the distance $r_{2}$, then insertion is optimally made by a tangential impulse at this point. If the apogee is at a distance slightly less than or greater than $r_{2}$, then a two-impulse insertion is required; but the increment in characteristic velocity is only of the order of $\left(\gamma_{f}\right) .^{2}$ Therefore, it is reasonable to consider only oneimpulse deorbit and one-impulse LEO insertion for the aeroassited coplanar transfer.

We now proceed to consider a less idealized aeroassisted orbit transfer in which the vehicle flies a skip trajectory through the atmosphere. Determining the minimum-fuel trajectory and control in this case requires the formulation and numerical solution of an optimization problem.

\section{Equations of Motion}

The equations of motion for planar atmospheric flight are ${ }^{4}$

$$
\begin{aligned}
& \frac{\mathrm{d} r}{\mathrm{~d} t}=V \sin \gamma \\
& \frac{\mathrm{d} V}{\mathrm{~d} t}=-\frac{\rho S C_{D} V^{2}}{2 m}-g \sin \gamma \\
& V \frac{\mathrm{d} \gamma}{\mathrm{d} t}=\frac{\rho S C_{L} V^{2}}{2 m}-\left(g-\frac{V^{2}}{r}\right) \cos \gamma
\end{aligned}
$$

assuming a nonrotating atmosphere. It is assumed that when $r>R$ the flight is Keplerian. Hence, we shall consider a Newtonian gravitational attraction, that is

$$
g=\mu / r^{2}
$$

Furthermore, it is assumed that the drag polar is parabolic, namely

$$
C_{D}=C_{D_{0}}+K C_{L}^{2}
$$

With this relation, using $C_{L}$ as a control corresponds physically to using pitch modulation to shape the trajectory. $C_{L}$ is allowed to assume both positive and negative values. A negative $C_{L}$ value can be interpreted as resulting either from a negative pitch angle or from a positive pitch angle with the vehicle flying upside down. It is convenient to use a nor-

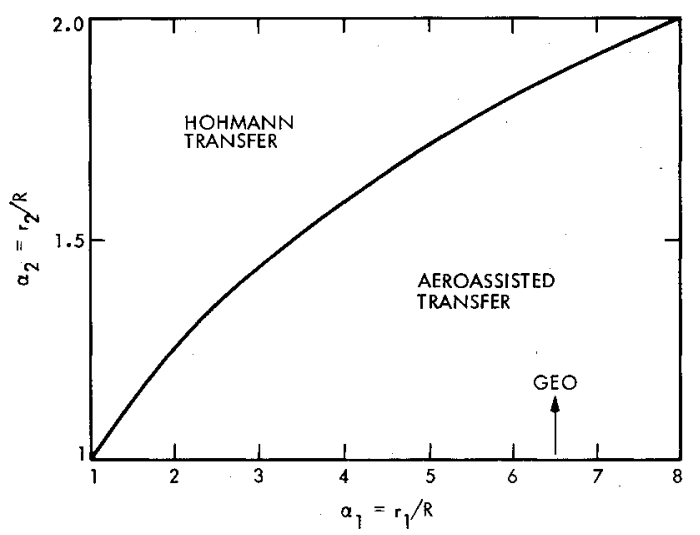

Fig. 2 Comparison of characteristic velocities for Hohmann and idealized aeroassisted transfers. malized lift control

$$
\lambda=C_{L} / C_{L}^{*}
$$

where $C_{L}^{*}$ is the lift coefficient corresponding to the maximum lift-to-drag ratio $E^{*}$. In tems of $C_{D_{o}}$ and $K$, we have

$$
C_{L}^{*}=\sqrt{C_{D_{o}} / K ;} \quad E^{*}=\frac{1}{2 \sqrt{K C_{D_{o}}}}
$$

Using the following dimensionless variables and parameters

$$
\begin{array}{clrl}
h=H / H_{e} ; & v=V / \sqrt{\mu / R} ; & \tau=\frac{t}{H_{e}} \sqrt{\mu / R} \\
\delta=\rho / \rho_{0} ; & b=R / H_{e} ; & B=\frac{\rho_{0} S H_{e} C_{L}^{*}}{2 m}
\end{array}
$$

the equations of motion can be rewritten as

$$
\begin{aligned}
& \frac{\mathrm{d} h}{\mathrm{~d} \tau}=v \sin \gamma \\
& \frac{\mathrm{d} v}{\mathrm{~d} \tau}=-\frac{B \delta}{2 E^{*}}\left(1+\lambda^{2}\right) v^{2}-\frac{b}{(b-1+h)^{2}} \sin \gamma \\
& \frac{\mathrm{d} \gamma}{\mathrm{d} \tau}=B \delta \lambda v+\frac{\cos \gamma}{(b-l+h)}\left[v-\frac{b}{(b-l+h) v}\right]
\end{aligned}
$$

Besides being preferable for numerical computation, the dimensionless equations of motion, Eq. (10), focus attention on the critical aerodynamic parameters which affect flight, namely, the lift loading coefficient $B$ and the maximum liftto-drag ratio $E^{*}$. Again $\lambda$ is the modulated lift control, scaled such that $\lambda=1$ corresponds to flight at the maximum lift-todrag ratio.

\section{The Optimization Problem}

The optimization problem is to find the values of the tangential $\Delta v_{l}$, a scalar parameter, and the lift control $\lambda$, a function of time, which minimize the total characteristic velocity

$$
\Delta v_{1}+\Delta v_{2}=\sqrt{1 / \alpha_{1}}-v_{e} \cos \gamma_{e} / \alpha_{1}+\sqrt{1 / \alpha_{2}}-v_{f} \cos \gamma_{f} / \alpha_{2}
$$

Equivalently, we can maximize the function

$$
J=v_{e} \cos \gamma_{e} / \alpha_{I}+v_{f} \cos \gamma_{f} / \alpha_{2}
$$

The atmospheric entry and exit variables must satisfy the relations

$$
\left(2-v_{e}^{2}\right) \alpha_{I}^{2}-2 \alpha_{I}+v_{e}^{2} \cos ^{2} \gamma_{e}=0
$$

and

$$
\left(2-v_{f}^{2}\right) \alpha_{2}^{2}-2 \alpha_{2}+v_{f}^{2} \cos ^{2} \gamma_{f}=0
$$

which are derived from the conservation of energy and angular momentum equations for the HEO-to-entry and exitto-LEO transfer orbits, respectively. At entry we have

$$
\tau_{e}=0 \text { and } h_{e}=I
$$

and at exit

$$
\tau_{f} \text { free and } h_{f}=I
$$

We now proceed to derive necessary conditions for the optimal solution. Introducing adjoint variables $p_{h}, p_{v}$, and 
$p_{\gamma}$, we form the Hamiltonian

$$
\begin{aligned}
\mathscr{K} & =p_{h} v \sin \gamma-p_{v}\left\{\frac{B \delta}{2 E^{*}} v^{2}\left(I+\lambda^{2}\right)+\frac{b \sin \gamma}{(b-I+h)^{2}}\right\} \\
& +p_{\gamma}\left\{B \delta v \lambda+\frac{\cos \gamma}{(b-1+h)}\left[v-\frac{b}{(b-1+h) v}\right]\right\}
\end{aligned}
$$

With respect to the lift control $\lambda, \mathcal{H}$ is maximized when

$$
\lambda=\frac{E^{*} p_{\gamma}}{v p_{v}}
$$

However, realistically, the range of values which $\lambda$ can assume is bounded; namely, the lift control must satisfy the inequality constraint

$$
|\lambda| \leq \lambda_{\max }
$$

where $\lambda_{\max }$ is a positive constant whose value is dictated by the aerodynamic characteristics of the vehicle. According to the Maximum Principle, we find that the optimal lift control is determined by the rule

$$
\lambda= \begin{cases}\lambda_{\max } & \text { when } \lambda \geq \lambda_{\max } \\ \Lambda & \text { when }|\lambda|<\lambda_{\max } \\ -\lambda_{\max } & \text { when } \lambda \leq-\lambda_{\max }\end{cases}
$$

where $\Lambda=E^{*} p_{\gamma} /\left(v p_{v}\right)$. In determining this rule, we have used the fact that the Hamiltonian is a quadratic function of $\lambda$ whose second derivative with respect to $\lambda$ is negative.

The adjoint variables satisfy the necessary conditions

$$
\frac{\mathrm{d} p_{h}}{\mathrm{~d} \tau}=-\frac{\partial \mathcal{H}}{\partial h} ; \frac{\mathrm{d} p_{v}}{\mathrm{~d} \tau}=-\frac{\partial \mathcal{H C}}{\partial v} ; \frac{\mathrm{d} p_{\gamma}}{\mathrm{d} \tau}=-\frac{\partial \mathcal{H C}}{\partial \gamma}
$$

However, note that to compute the optimal control only the value of $\Lambda$ is required. This suggests replacing the three differential equations, Eqs. (21), with two which involve only ratios of the adjoint variables, namely $\Lambda$ and $F=p_{h} / p_{\nu}$. Differentiating the expresions for $\Lambda$ and $F$ with respect to time and using Eqs. (21), we obtain

$$
\begin{aligned}
& \frac{\mathrm{d} \Lambda}{\mathrm{d} \tau}=-E^{*} F \cos \gamma+\frac{\cos \gamma}{(b-1+h)^{2} v}\left[\frac{2 b \Lambda^{2}}{E^{*}}+b E^{*}\right] \\
& +\frac{\Lambda \sin \gamma}{(b-1+h)}\left[v+\frac{b}{(b-1+h) v}\right]
\end{aligned}
$$

and

$$
\begin{aligned}
\frac{\mathrm{d} F}{\mathrm{~d} \tau} & =-\frac{2 b \sin \gamma}{(b-I+h)}+\frac{v \Lambda \cos \gamma}{E^{*}(b-I+h)^{2}}\left[v-\frac{2 b}{(b-I+h) v}\right] \\
& +\frac{B v^{2}}{2 E^{*}}\left(I+\lambda^{2}\right) \delta^{\prime}-\frac{v^{2}}{E^{*}} \Lambda B \lambda \delta^{\prime}+F^{2} \sin \gamma-\frac{F B}{E^{*}} \delta v\left(1+\lambda^{2}\right) \\
& +\frac{v}{E^{*}} \Lambda F B \delta \lambda+\frac{\Lambda F \cos \gamma}{E^{*}(b-1+h)}\left[v+\frac{b}{(b-I+h) v}\right]
\end{aligned}
$$

where

$$
\delta^{\prime}=\frac{\mathrm{d} \delta}{\mathrm{d} h}=\frac{H_{e}}{\rho_{0}} \frac{\mathrm{d} \rho}{\mathrm{d} H}
$$

Writing the modified Hamiltonian in terms of $\Lambda$ and $F$, we have

$$
\begin{aligned}
\overline{\mathfrak{F}} & =F \sin \gamma-\frac{B}{2 E^{*}} \delta v\left(I+\lambda^{2}\right)-\frac{b \sin \gamma}{(b-I+h)^{2} v} \\
& +\frac{\Lambda B \delta v \lambda}{E^{*}}+\frac{\Lambda \cos \gamma}{E^{*}(b-I+h)}\left[v-\frac{b}{(b-I+h) v}\right]
\end{aligned}
$$

Since the equations of motion, Eqs. (10), do not depend explicitly on time and the final time is not prescribed, we have the Hamilton integral

$$
\overline{\mathfrak{H}}=0
$$

Now, rather than the original six differential equations, we have five, namely, Eqs. (10) for the three states, and Eqs. (22) and (23) for $\Lambda$ and $F$. Integation of these equations will yield extremal trajectories for a number of problems which differ only in the entry and exit conditions which must be satisfied. Besides having reduced the dimension from six to five, this

\begin{tabular}{|c|c|c|c|c|c|}
\hline \multirow[t]{2}{*}{. } & \multicolumn{3}{|c|}{$L / D$ capability } & \multicolumn{2}{|c|}{ Heating rate } \\
\hline & Low & Moderate & High & Unconstrained & Constrained \\
\hline$(L / D)_{\max }$ & 0.845 & 1.5 & 2.9 & 1.5 & 1.5 \\
\hline Target perigee $r_{p}, \mathrm{~km}$ & 6400.0 & 6400.0 & 6400.0 & 6415.0 & 6415.0 \\
\hline$\Lambda_{e}\left(\Lambda_{p}\right)$ & 2.303247 & 2.701724 & 3.2586836 & 2.947660826 & $6.56(.79867315)$ \\
\hline Exit flight path angle, deg & 0.3 & 0.4 & 0.3 & 0.45 & 0.49 \\
\hline LEO orbit radius, $\mathrm{km}$ & 6558.8 & 6578.7 & 6557.6 & 6608.0 & 6625.0 \\
\hline$\Delta V_{2}, \mathrm{~m} / \mathrm{s}$ & 26.1 & 31.0 & 25.2 & 40.5 & 45.1 \\
\hline Ideal $\Delta V_{2}$ for same $\mathrm{LEO}, \mathrm{m} / \mathrm{s}$ & 18.3 & 24.0 & 18.0 & 32.6 & 37.7 \\
\hline Min altitude, $\mathrm{km}$ & 58.8 & 58.2 & 51.5 & 61.2 & 64.0 \\
\hline Max dynamic pressure, $\mathrm{kN} / \mathrm{m}^{2}$ & 15.9 & 18.6 & 44.2 & 13.1 & 8.8 \\
\hline $\begin{array}{l}\text { Max convective heating rate } \\
\text { for a one meter sphere, } \mathrm{W} / \mathrm{cm}^{2}\end{array}$ & 193.1 & 222.8 & 361.4 & 190.8 & 150.0 \\
\hline Max g's & 3.6 & 2.7 & 1.8 & 1.9 & 3.7 \\
\hline
\end{tabular}
formulation has the distinct advantage that four of the five dependent variables are physical variables. (Actually, $\Lambda$ has physical meaning only if $|\Lambda| \leq \lambda_{\max }$.) This situation eases the difficulty in guessing unknown initial conditions during the course of solving the boundary value problem. The nonphysical variable $F$ can almost always $(\sin \gamma \neq 0$ ) be computed from the other four using the Hamiltonian integral, Eq. (24). Indeed, one might use the Hamiltonian integral to eliminate the need for solving the differential equation for $F$. However, to avoid the difficulty in evaluating $F$ at the singularity, $\sin \gamma=0$, we shall integrate the equation for $F$, and instead use the Hamiltonian integral as a check on the accuracy of the numerical integration.

Table 1 Characteristics of minimum-fuel trajectories 
The heating rate, $H_{R}$, along the atmospheric trajectory, is computed according to the equation

$$
H_{R}=\left(3.08 \times 10^{-4}\right) \rho^{1 / 2} V^{3.08}
$$

where $\rho$ is the atmospheric density in $\mathrm{kg} / \mathrm{km}^{3}$ and $V$ is the speed in $\mathrm{km} / \mathrm{s}$. Equation (26) gives the convective heating rate for a sphere with a radius of $1 \mathrm{~m}$, under conditions of laminar flow. Since only relative changes are of concern, this model will suffice.

\section{Method of Numerical Solution}

We shall only concern ourselves with minimizing $\Delta V_{2}$. Although $\Delta V_{I}$ is the larger of the two burns, for the HEO considered the difference between the value of $\Delta V_{1}$, required to target to a perigee at the atmospheric boundary, $R=6498$ $\mathrm{km}$, and that required to target to a perigee at the surface of the Earth is a mere $13 \mathrm{~m} / \mathrm{s}$. In contrast, $\Delta V_{2}$ is very sensitive to the values of the exit parameters $v_{f}$ and $\gamma_{f}$. For example, the $\Delta V_{2}$ required for a given HEO to LEO transfer can increase by $100 \mathrm{~m} / \mathrm{s}$ or more for each degree above zero in the exit flight path angle, $\dot{\gamma}_{f}$.

Knowing that a skip trajectory with $\gamma_{f}=0$ deg leads to the minimum $\Delta V_{2}$ at the LEO to which the ascending orbit is tangent, we employed the following approach to compute minimum-fuel trajectories and controls. A target perigee, $\dot{r}_{p}$, is chosen and from this the entry parameters $v_{e}$ and $\gamma_{e}$ are determined according to the equations

$$
\begin{gathered}
v_{e}^{2}=2\left[1-1 /\left(\alpha_{l}+\alpha_{p}\right)\right] \\
\cos ^{2} \gamma_{e}=\left[2 \alpha_{1}-\left(2-v_{e}^{2}\right) \alpha_{l}^{2}\right] / v_{e}^{2}
\end{gathered}
$$

Then, using the computed values of $v_{e}$ and $\gamma_{e}, h_{e}=1$, and a pair $\left(\Lambda_{e}, F_{e}\right)$ as initial conditions, Eqs. (10), (22), and (23) are integrated from $\tau=0$ to $h=1$, using Eq. (20) to determine the lift control. The pair $\left(\Lambda_{e}, F_{e}\right)$ is determined by choosing $\Lambda_{e}$ and using Eq. (24) to solve for the corresponding value of $F_{e}$, with $v, \gamma$, and $h$ as specified above. The integration is performed by a variable order, linear, multistep predictorcorrector routine of the Adams-Moulton type, ${ }^{5}$ with the local absolute error controlled to less than $1.0 \times 10^{-8}$ for each of the five dependent variables. In all cases studied, it has been possible to find a value of $\Lambda_{e}$ such that $\gamma_{f}=0 \mathrm{deg}$ at exit by iterative search. The corresponding value of $v_{f}$ determines the apogee of the transfer orbit following exit, and hence, the LEO to which the vehicle is optimally transferred. As the value of $r_{p}$ is lowered from $R, \gamma_{f}=0 \mathrm{deg}$ continues to be reachable, but the exit speed decreases, resulting in lower LEO transfers. There is a certain critical value of $r_{p}$ below which the lifting capability of the vehicle is insufficient to effect a skip trajectory. However, for the vehicle studied in the following, the range of reachable LEO's extended all the way down to altitudes of less than $200 \mathrm{~km}$.

A trajectory and control computed in this manner are optimal in the following sense. First, the necessary conditions, [Eqs. (10), (22), and (23)], the entry and exit conditions [Eqs. (15) and (16)], and Eqs. (13) and (14) are satisfied. Second, the lift control satisfies the constraint [Eqs. (19)] Third, the near-zero degree flight path angle at exit ensures that the circularizing $\Delta V_{2}$ to achieve the LEO to which the post-exit orbit is tangent is the absolute minimum, when compared to those for all other aeroassisted transfers from the same HEO to the same LEO. (The exit flight path angles achieved, as indicated in Table 1, are a few tenths of a degree. The iteration on $\Lambda_{e}$ was stopped at this point because the associated value of $\Delta V_{2}$ was within $8 \mathrm{~m} / \mathrm{s}$ of the lower bound set by the idealized transfer.) Fourth, although only $\Delta V_{2}$ has been minimized, the characteristic velocity, $\Delta V_{1}+\Delta V_{2}$, is very close to the absolute minimum. The value of $\Delta V_{1}$ for the cases shown in Table 1 is within $10.4 \mathrm{~m} / \mathrm{s}$ of the lower bound on $\Delta V$, given by the idealized transfer. Thus, the characteristic velocity cannot get much smaller. Equally important, however, is whether or not the trajectory and/or control would change significantly if the characteristic velocity were reduced the last few meters per second. Numerical experience indicates that they do not. As the exit flight path angle is reduced, the atmospheric trajectory changes very little. Indeed, the value of $\Lambda_{e}$ is being changed only slightly (parts in $10^{6}$ or less) to get the exit angle below a few tenths of a degree. This level of change in $\Lambda_{e}$ affects most of the trajectory almost negligibly, but extends the trajectory in order to achieve the lower exit angle. Furthermore, as mentioned above for zero exit flight path angle atmospheric trajectories, there is a one-to-one mapping, based on numerical experience, from values of $r_{p}$ to values of $v_{f}$, and hence, to the LEO's for which the $\Delta V_{2}$ is a minimum. Therefore, if $r_{p}$ were increased in order to decrease $\Delta V_{1}$, the corresponding $\Delta V_{2}$ would be greater. Given the low sensitivity of $\Delta V_{l}$ to changes in the value of $r_{p}$, it is unlikely that the characteristic velocity could be reduced much, if any, by adjusting $r_{p}$. In conclusion, a trajectory and control solution computed in the manner described above is a realistic approximation to that which gives the absolute minimum characteristic velocity, and henceforth we shall refer to such a solution as a minimumfuel solution.

When a heat rate constraint is imposed, the solution procedure is somewhat different. We follow an approach used in Ref. 6. The heating rate, for a skip trajectory, reaches its maximum value shortly after entry, in a monotonic fashion. It then decreases during the remainder of the flight, although some oscillation may occur. In order to satisfy a heating rate constraint, $H_{R} \leq\left(H_{R}\right)_{\max }$, we shall assume that it is sufficient to control the first peak of the heating rate function, such that the peak value is equal to $\left(H_{R}\right)_{\max }$. Furthermore, we shall assume that, once this peak value is reached, flight does not continue on the heating constraint boundary. These assumptions allow us to solve the constrained problem in two stages, each requiring an iteration on only one parameter.

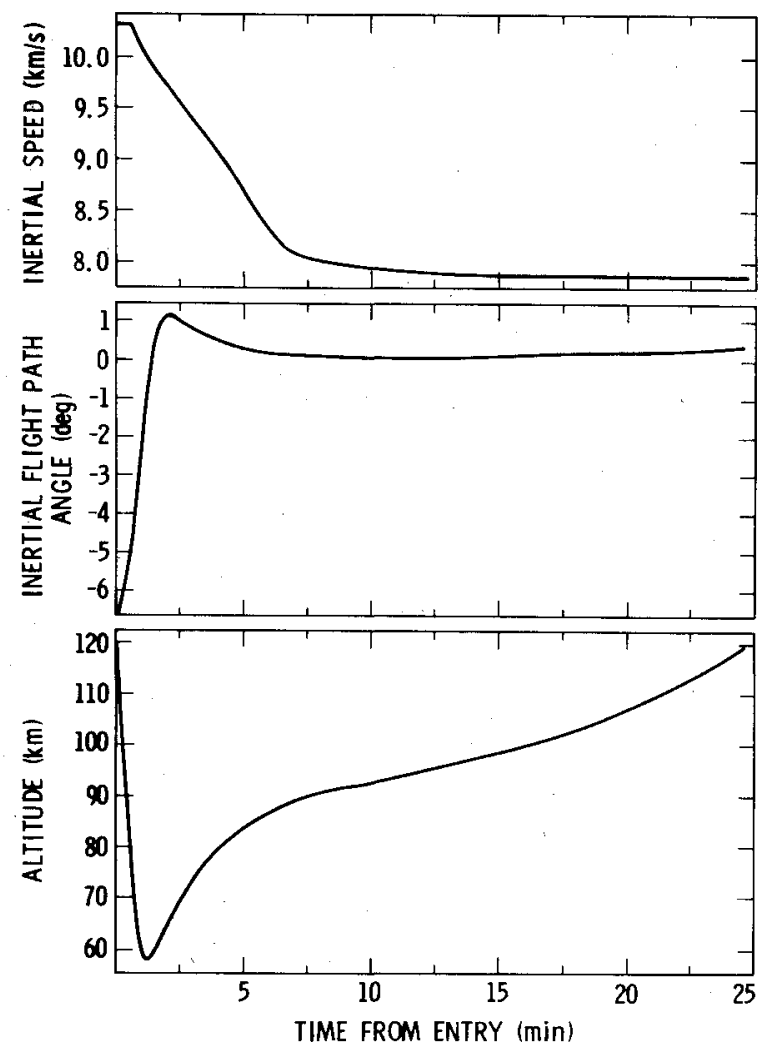

Fig. 3 Time histories of state variables, $(L / D)_{\max }=1.5$. 
In the first stage, we begin at $\tau=0$ as in the unconstrained case, except that now the goal is to choose $\Lambda_{e}$ such that $H_{R}=\left(H_{R}\right)_{\max }$ at the time when the derivative of the heating rate with respect to time is equal to zero. Once this value of $\Lambda_{e}$ is found the minimum-fuel trajectory up to the peak heating rate is determined. The second stage is to find a value for $\Lambda$, $\Lambda=\Lambda_{p}$, such that, when Eqs. (10), (22), and (23) are integrated from the time of the peak heating rate to atmospheric exit, the value of the exit flight path angle is zero degrees. Indeed, in the cases studied, it has been possible to find such values of $\Lambda_{e}$ and $\Lambda_{p}$. Thus, while the functions $\Lambda$ and $F$ are, in general, discontinuous at the time of the peak heating rate, the states $h, v$, and $\gamma$ are always continuous.

\section{Minimum-Fuel Trajectories}

For all the cases reported below, the transfer is from goestationary Earth orbit (GEO), for which $r_{l}=42,241 \mathrm{~km}$. The radius of the atmosphere is $6498 \mathrm{~km}$. Above this distance, the density is identically zero. Over the altitudes of atmospheric flight, $40-120 \mathrm{~km}$ (where the radius of the Earth is taken to be $6378 \mathrm{~km}$ ), the density is approximated by a fifthdegree Chebyshev polynomial whose coefficients were determined by a least-squares fit to the U.S. Standard Atmosphere, 1976 (Ref. 7). The vehicle mass-to-surface area is $300 \mathrm{~kg} / \mathrm{m}^{2}$ for all cases.

Concerning the radius of the atmosphere, a few remarks are in order. Since the atmospheric density variation with altitude is exponential in nature, according to the U.S. Standard Atmosphere, the interface between the atmosphere and the vacuum is not well defined. It is common practice to choose the entry (and exit) radius such that the aerodynamic force produces a slight but detectable acceleration to modify the Keplerian orbit. Thus, the critical parameter in determining the radius value is the lift loading coefficient $B$. Accordingly, the value of $6498 \mathrm{~km}$ for the radius is consistent with the values of $B$ used for numerical computations in this study. With regard to the optimization results, the characteristic velocity for the aeroassisted transfer shows little sensitivity to the value of $R$. For the GEO to $350 \mathrm{~km}$ altitude circular LEO transfer, changing the value of $R$ by $10 \mathrm{~km}$ in either direction alters the characteristic velocity by less than $3 \mathrm{~m} / \mathrm{s}$.

\section{Unconstrained}

We begin by presenting some minimum-fuel trajectories under conditions of unbounded lift and unconstrained heating rate. Three vehicle configurations were considered, as distinguished by their respective maximum $L / D$ capabilities, namely, $0.845,1.5$, and 2.9. Data from wind tunnel tests is available for vehicles with these maximum $L / D$ capabilities (Refs. 8, 9, and 10, respectively) and the values for the parameters $C_{D_{o}}$ and $K$ which appear in the parabolic drag polar were chosen to best fit the data. The values used for the pair $\left(C_{D_{0}}, K\right)$ were $(0.21,1.67),(0.10,1.11)$, and $(0.017$, $1.76)$, respectively.

Note that although constant values for $C_{D_{o}}$ and $K$ are used here for the numerical computations, the preceding theory is more generally applicable. For precise computation of an optimal trajectory, it is necessary to model the coefficients $C_{D_{0}}$ and $K$ as continuous functions of the altitude $h$ and the speed $v$ since at low altitude and low hypersonic speed these coefficients are functions of the Mach number and the Reynolds number. In Eqs. (10), we then have $B$ and $E^{*}$ as functions of $h$ and $v$. The Hamiltonian integral Eq. (25), with $\mathcal{H C}$ as given in Eq. (24), remains unchanged. The use of the adjoint ratios $\Lambda$ and $F$ is still valid. The only change is that in Eqs. (22) and (23), we would have additional terms containing the nonvanishing partial derivatives $B_{h}$ and $E^{*}{ }_{h}$, with respect to $h$, and $B_{v}$ and $E_{v}^{*}$, with respect to $v$. The method of numerical solution remains applicable and involves no additional difficulty. Examples of modeling the aerodynamic coefficients as functions can be found in Ref. 4. For our purposes here, it suffices to use the best fit constant values for
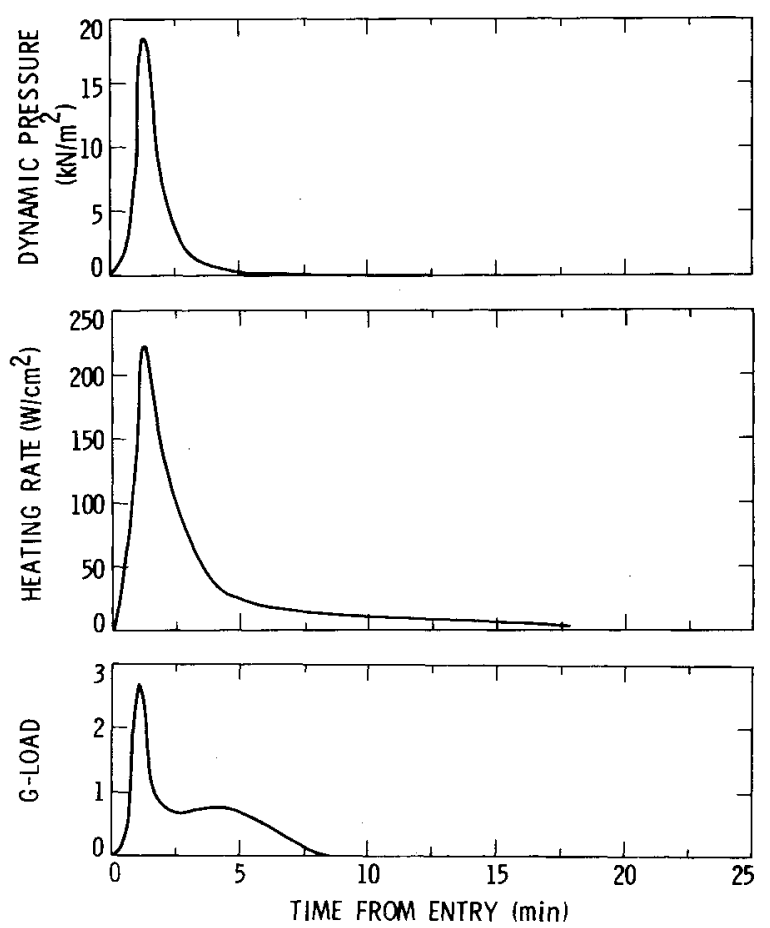

Fig. 4 Time histories of heating rate, dynamic pressure, and $g$ load, $(L / D)_{\max }=1.5$.

$C_{D_{o}}$ and $K$ in the altitude range of effective aerodynamic maneuvering. Since the aeroassisted coplanar transfer requires only energy depletion, it is reasonable to expect that a more precise treatment of the vehicle aerodynamics would not drastically alter the characteristic behavior of the lift control or the resulting performance.

For each of the three maximum $L / D$ cases, we have fixed $r_{p}=6400 \mathrm{~km}$ and have searched and found the $\Lambda_{e}$ such that $\gamma_{f}=0$ deg. In this manner, a minimum-fuel trajectory for each case was generated. The corresponding LEO orbits, to which the transfers are optimal, are not exactly the same, but are close enough to permit comparisons. The alternative approach of specifying the LEO orbit a priori would require seaching on two parameters, $r_{p}$ and $\Lambda_{e}$, in order to determine the minimum-fuel trajectory.

Certain characteristics of the minimum-fuel unconstrained trajectories are given in the first three columns of Table 1 . We see that the high $L / D$ vehicle penetrates farthest into the atmosphere and experiences the highest dynamic pressure and heating rate. The low $L / D$ vehicle experiences the highest $g$ load. For comparison, the shuttle design limits for dynamic pressure and $g$-load are $16 \mathrm{kN} / \mathrm{m}^{2}$ and 2.5 , respectively. ${ }^{11}$ Time histories of the state variables for the $(L / D)_{\max }=1.5$ case are shwon in Fig. 3; those for the heating rate, dynamic pressure, and $g$ load are shown in Fig. 4. The behaviors illustrated in these two figures are qualitatively representative of all the cases discussed in this paper.

Figure 5 shows the lift-to-drag ratio as a function of the time from atmospheric entry for the three cases. A similar pattern is followed in each case. The maximum positive $L / D$ is used initially to recover from the downward plunge. As the flight path angle becomes positive, the maximum negative $L / D$ is used to level off the flight. These first two phases occur within the first four minutes of flight. Of course, although the basic pattern is similar, quantitatively there are definite differences in the flight characteristics of the three $L / D$ vehicles, as indicated in Table 1 . After the first four minutes, a negative $L / D$ is used to maintain flight at a small positive flight path angle in order to achieve the desired shallow exit. The required negative $L / D$ increases as the flight proceeds to compensate for the decreasing atmospheric density. 


\section{Bounded Lift}

For the vehicle, with $(L / D)_{\max }=1.5$, wind tunnel data show that the lift coefficient does not exceed 0.9 in absolute value. Thus, we impose the constraint

$$
\left|C_{L}\right| \leq 0.9
$$

which corresponds to setting $\lambda_{\max }=3.0$ in Eq. (20). The resulting lift control is given by the dashed curve in Fig. 6 . For comparison, the corresponding curve with $C_{L}$ unbounded is given by the solid curve. We see that flight is along the constraint boundary for much of the flight. The important point, however, is that a near-zero exit flight path angle is still reachable by proper choice of $\Lambda_{e}$.

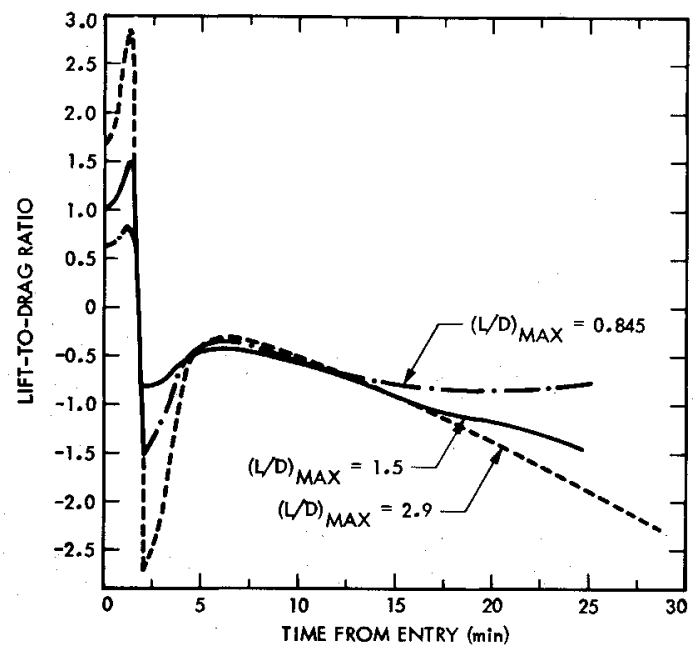

Fig. 5 Time histories of lift-to-drag ratio.

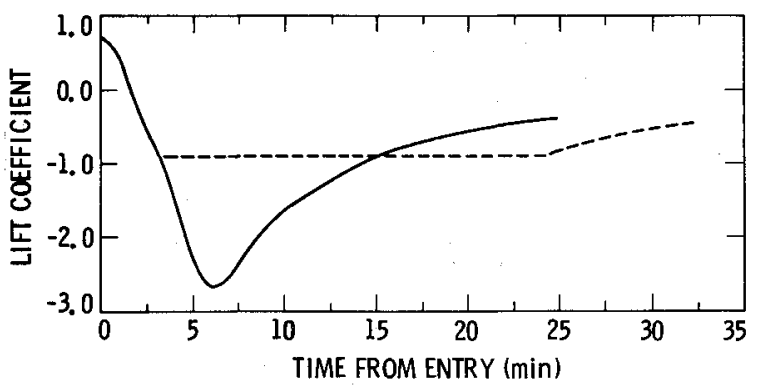

Fig. 6 Time histories of optimal lift control for unbounded and bounded cases.

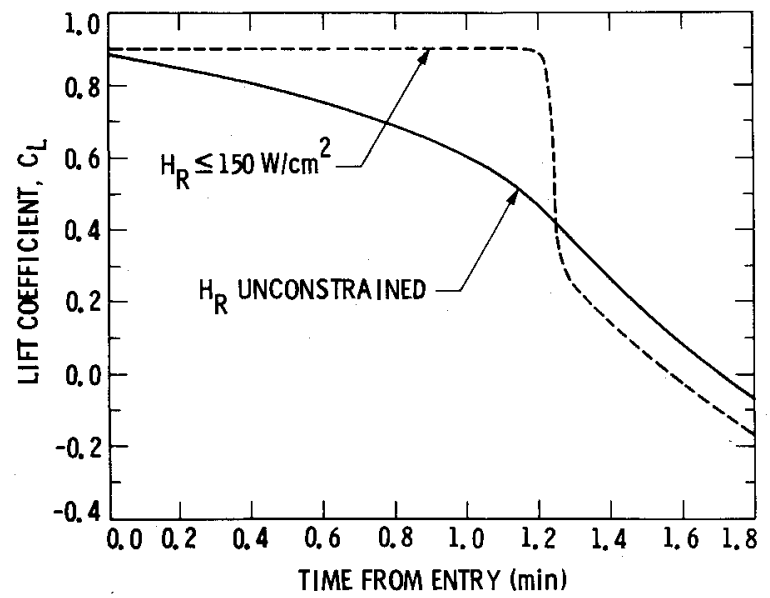

Fig. 7 Time histories of optimal lift control for unconstrained and constrained heating rate cases (only first two minutes shown).

\section{Constrained Heating Rate}

Using Eq. (26), the heating rate along the minimum-fuel trajectory can be calculated. Referring to this as the unconstrained heating rate, we can ask the question: What is the minimum-fuel trajectory if the maximum heating rate is constrained to be no greater than some fraction of the maximum unconstrained heating rate?

In order to see the effect of a heating rate constraint, we again consider the configuration with $(L / D)_{\max }=1.5$, as described earlier, except that the target perigee is taken to be $6415 \mathrm{~km}$. The minimum $\Delta V_{2}$ trajectory is computed first with the heating rate unconstrained. The maximum heating rate is found to be $190.8 \mathrm{~W} / \mathrm{cm}^{2}$ for a reference $1 \mathrm{~m}$ sphere. Next, the minimum-fuel trajectory is computed with all conditions identical, except that the heating rate is constrained not to exceed $150.0 \mathrm{~W} / \mathrm{cm}^{2}$. In both cases, a the lift coefficient is bounded, as described earlier.

Certain characteristics of the unconstrained and constrained cases are given for comparison in the last two columns of Table 1 . In both cases, a near-zero exit flight path angle is reachable and the $\Delta V_{2}$ is within $8 \mathrm{~m} / \mathrm{s}$ of that for the idealized transfer. With the heating rate constrained, the vehicle does not penetrate the atmosphere as deeply; the maximum dynamic pressure is reduced; but the maximum $g$ load is increased.

The optimal lift control, for each case, is plotted vs time in Fig. 7. We see that the vehicle flies initially at $\left(C_{L}\right)_{\max }$ in the constrained case; whereas in the unconstrained case, $C_{L}$ is decreasing steadily during the same period. By flying at $\left(C_{L}\right)_{\max }$ initially, and correspondingly at a higher $C_{D}$, the vehicle slows down higher in the atmosphere, allowing recovery from the downward plunge, which occurs subsequently at the maximum positive $L / D$, to take place at a lower atmospheric density, or equivalently, at a higher altitude. In this manner, higher heating rates are avoided.

As a final note, the minimum entry flight path angle from which the vehicle can recover and achieve the prescribed exit state conditions, is raised when a heating rate constraint is imposed (that is, raised with respect to the unconstrained case). The reason is that if the entry is too steep, excessive heating rates cannot be avoided, even by flying at the maximum positive $C_{L}$. In the particular case investigated here, an optimal solution was found for entry angles as low as $-6.5^{\circ}\left(r_{p}=6400 \mathrm{~km}\right)$ in the unconstrained case. In the constrained case, the lowest entry angle that could be tolerated was $-6.0^{\circ}\left(r_{p}=6415 \mathrm{~km}\right)$.

\section{Summary and Conclusions}

Under the given assumptions and restrictions, minimumfuel aeroassisted coplanar transfer from high orbit to low orbit using lift modulation has been considered. An idealized version of the transfer lent itself to analytic treatment and allowed a lower bound on the characteristic velocity for any given HEO to LEO aeroassisted transfer to be determined. In order to examine minimum-fuel transfer under more realistic conditions, an optimization problem was formulated and solved numerically. It was found that for each given HEO to LEO transfer considered, even with bounded lift and/or a heating rate constraint, a characteristic velocity within 10 $20 \mathrm{~m} / \mathrm{s}$ of the lower bound is achievable. The optimal aeroassisted mode of transfer requires less fuel than the optimal all-propulsive mode for a wide range of high orbit to low orbit transfers. For the transfer from GEO to a circular $350 \mathrm{~km}$ altitude orbit, the characteristic velocity is $2.32 \mathrm{~km} / \mathrm{s}$ less for the aeroassisted mode.

The characteristic lift program for the atmospheric portion of the minimum-fuel transfer is to fly at the maximum positive $L / D$ initially to recover from the downward plunge, and then to fly at negative $L / D$ to level off the flight such that the vehicle skips out of the atmosphere with a flight path angle near zero degrees. This program is modified at the beginning if high heating rates are to be avoided. Flight, 
initially at maximum lift, and, correspondingly at high drag, lowers the vehicle's speed higher in the atmosphere, allowing recovery from the downward plunge (which occurs subsequently using the maximum positive $L / D$ ) to take place at a lower atmospheric density, or equivalently, at a higher altitude.

\section{Acknowledgment}

The research described in this paper was carried out by the Jet Propulsion Laboratory, California Institute of Technology, under contract with the National Aeronautics and Space Administration. The authors thank E.A. Rinderle for developing the software used for the numerical computations.

\section{References}

${ }^{1}$ Cruz, M.I., French, J.R., and Austin, R.E., "System Design Concepts and Requirements for Aeroassisted Orbital Transfer Vehicles,"' AIAA Paper 82-1379, Aug. 1982.

${ }^{2}$ Walberg, G.D., "A Review of Aeroassisted Orbit Transfer," AIAA Paper 82-1378, Aug. 1982.

${ }^{3}$ Buseman, A. and Vinh, N.X., "Optimum Constrained Disorbit by Multiple Impulses," Journal of Optimization Theory and Applications, Vol. 2, No. 1, 1968, pp. 40-64.
${ }^{4}$ Vinh, N.X., Optimal Trajectories in Atmospheric Flight, Elsevier Scientific Publishing Company, New York, 1981.

${ }^{5}$ Krogh, F.T., "VODQ/SVDQ/DVDQ—Variable Order Integrators for the Numerical Solution of Ordinary Differential Equations," JPL Technical Utilization Document No. CP-2308, NPO-11643, May 1969.

${ }^{6}$ Chern, J.S., Yang, C.S., Vinh, N.X., and Hwang, G.R., “Optimal Three-Dimensional Reentry Trajectories Subject to Deceleration and Heating Constraints," Paper No. 82-309, 33rd Congress of the International Astronautical Federation, Paris, France, Sept. 1982.

7 “'U.S. Standard Atmosphere, 1976," NOAA-S/T 76-1562, U.S. Government Printing Office, Washington, D.C.

${ }^{8}$ Penland, J.A., "A Study of the Stability and Location of the Center of Pressure on Sharp, Right Circular Cones at Hypersonic Speeds," NASA TN D-2283, 1964.

${ }^{9}$ Miller, C.G. and Gnoffo, P.A., "An Experimental Investigation of Hypersonic Flow Over Biconics at Incidence and Comparison to Prediction," AIAA Paper 82-1382, Aug. 1982.

${ }^{10}$ Books, C.W., Jr. and Cone, D., Jr., "Hypersonic Aerodynamic Characteristics of Aircraft Configurations with Canard Controls," NASA TN D-3374, 1966.

${ }^{11}$ Harpold, J.C. and Graves, C.A., Jr., "'Shuttle Entry Guidance," Journal of the Astronautical Sciences, Vol. 27, No. 3, July 1979, pp. 239-268.

\title{
From the AIAA Progress in Astronautics and Aeronautics Series ...
}

\section{INTERIOR BALLISTICS OF GUNS-v. 66}

\author{
Edited by Herman Krier, University of Illinois at Urbana-Champaign, \\ and Martin Summerfield, New York University
}

In planning this new volume of the Series, the volume editors were motivated by the realization that, although the science of interior ballistics has advanced markedly in the past three decades and especially in the decade since 1970, there exists no systematic textbook or monograph today that covers the new and important developments. This volume, composed entirely of chapters written specially to fill this gap by authors invited for their particular expert knowledge, was therefore planned in part as a textbook, with systematic coverage of the field as seen by the editors.

Three new factors have entered ballistic theory during the past decade, each it so happened from a stream of science not directly related to interior ballistics. First and foremost was the detailed treatment of the combustion phase of the ballistic cycle, including the details of localized ignition and flame spreading, a method of analysis drawn largely from rocket propulsion theory. The second was the formulation of the dynamical fluid-flow equations in two-phase flow form with appropriate relations for the interactions of the two phases. The third is what made it possible to incorporate the first two factors, namely, the use of advanced computers to solve the partial differential equations describing the nonsteady twophase burning fluid-flow system.

The book is not restricted to theoretical developments alone. Attention is given to many of today's practical questions, particularly as those questions are illuminated by the newly developed theoretical methods. It will be seen in several of the articles that many pathologies of interior ballistics, hitherto called practical problems and relegated to empirical description and treatment, are yielding to theoretical analysis by means of the newer methods of interior ballistics. In this way, the book constitutes a combined treatment of theroy and practice. It is the belief of the editors that applied scientists in many fields will find material of interest in this volume. 